\title{
Carrier Transport across a Few Grain Boundaries in Highly Doped Polycrystalline Silicon
}

\author{
Yoshikazu Furuta ${ }^{1,4, *}$, Hiroshi Mizuta ${ }^{1,4}$, Kazuo NAKAZATO ${ }^{1,4}$, Yong T. TAN ${ }^{2,4}$, Toshio KAmiYA ${ }^{2,4}$, \\ Zahid A. K. DURRAnI ${ }^{2,4}$, Haroon AhMED ${ }^{2,4}$ and Kenji TANIGUCHI ${ }^{3}$ \\ ${ }^{1}$ Hitachi Cambridge Laboratory, Madingley Road, Cambridge CB3 OHE, U. K. \\ ${ }^{2}$ Microelectronics Research Centre, Cavendish Laboratory, University of Cambridge, Madingley Road, Cambridge CB3 0HE, U. K. \\ ${ }^{3}$ Electronics, Information Systems and Energy Engineering, Osaka University, 2-1 Yamada-oka, Suita, Osaka 565-0871, Japan \\ ${ }^{4}$ CREST JST (Japan Science and Technology), Shibuya TK Bldg., 3-13-11 Shibuya, Shibuya-ku, Tokyo 150-0002, Japan
}

(Received January 15, 2001; accepted for publication April 16, 2001)

We have fabricated nanometer-scale point-contact devices in 50-nm-thick poly-Si films with a grain size of from $150 \mathrm{~nm}$ to $20 \mathrm{~nm}$. Both linear and nonlinear $I_{\mathrm{ds}}-V_{\mathrm{ds}}$ characteristics were observed in these devices, corresponding to a channel without a grain boundary $(\mathrm{GB})$ and that with a single or a few GBs, respectively. The temperature dependence of resistivity indicated that the effective potential barrier height $q V_{\mathrm{B}}$ of the GBs for the devices which show the nonlinear $I_{\mathrm{ds}}-V_{\mathrm{ds}}$ characteristics ranges from $30 \mathrm{meV}$ to $80 \mathrm{meV}$. We discussed percolation conduction of electrons through a few GBs due to nonuniform GB properties in heavily doped poly-Si films.

KEYWORDS: polycrystalline silicon, electron transport, grain boundary, point-contact device, potential barrier height, intergrain

Polycrystalline silicon (poly-Si) has been widely studied for various applications such as thin film transistors, static random access memories (SRAMs) and stacked memory cells. $^{1,2)}$ The electrical properties of poly-Si films are sensitive to the process conditions, particularly those of crystallization and passivation methods. Significant efforts have been made to increase the carrier mobility of these films since this limits the ON current of poly-Si devices. Thin poly-Si films have also been studied as a building block for single-electron memories ${ }^{3,4)}$ where individual silicon grains are used as charging islands. In this application, poly-Si films with a grain size of less than $10 \mathrm{~nm}$ are used, such that the single-electron charging energy is sufficiently large to observe Coulomb blockade effects.

Despite these intensive studies, the relationship between the microscopic properties of an individual grain boundary (GB) and the macroscopic electric properties of poly-Si films is not clear. Very recently, a detailed investigation has been reported for $1-\mu \mathrm{m}$-thick poly-Si films with a grain size of about $0.3 \mu \mathrm{m} .{ }^{5)}$ In this study, electron transport through a small number of GBs has been measured for the first time by using a four-point probe technique, and the distribution of individual GB potential barrier height has been investigated. It has been shown that for low-doped devices with a donor concentration of $1 \times 10^{17} \mathrm{~cm}^{-3}$, the GB potential barrier height varies largely among devices, and this has been attributed to nonuniformity of potential barrier heights of GBs.

In this paper, we focus on the relationship between the local structure and transport properties of heavily-doped polySi films with a much smaller film thickness and grain size. By fabricating novel ultrashort point-contact devices where both the length and width of the channel are as small as the grain size, we study electron transport through a single or a few GBs in the channel. The main objectives of this study are to investigate the transport properties of a single GB and to establish "GB engineering" techniques.

Highly doped poly-Si films were prepared as follows: A 40-nm-thick $\mathrm{SiO}_{2}$ layer was thermally grown on a (100)oriented lightly-doped silicon substrate. An undoped 50-nm- thick amorphous silicon (a-Si) film was then deposited using low-pressure chemical vapor deposition (LPCVD) at $550^{\circ} \mathrm{C}$, followed by phosphorus ion implantation into the a-Si film layer with an energy of $20 \mathrm{keV}$ at a dose of $3 \times 10^{14} \mathrm{~cm}^{-2}$. Solid-phase crystallization (SPC) of the a-Si films was performed at $850^{\circ} \mathrm{C}$ for $30 \mathrm{~min}$ in an Ar ambient, crystallizing the films as well as activating the dopants electrically. By using a simulator ATHENA, ${ }^{6}$ the doping concentration in the poly-Si film was estimated to be as high as $10^{20} \mathrm{~cm}^{-3}$.

Side-gated point-contact device structures (see Fig. 1(a)) were defined by using a conventional electron-beam lithography technique with polymethylmethacrylate (PMMA) resist. The devices were electrically isolated by reactive ion etching into the silicon substrate with a mixture of $\mathrm{SiCl}_{4}$ and $\mathrm{CF}_{4}$. Figure 1(b) shows a scanning electron microscope (SEM) image of a Secco-etched ${ }^{7)}$ SPC thin poly-Si film. The grain size of the films after SPC ranges from $20 \mathrm{~nm}$ to $150 \mathrm{~nm}$, which is consistent with the cross-sectional transmission electron microscope (XTEM) observation. The XTEM observation
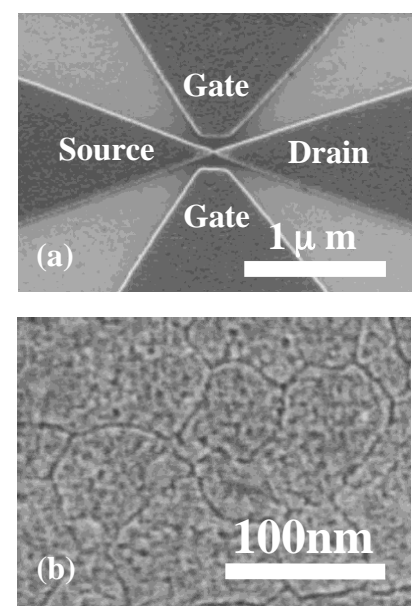

Fig. 1. (a) Scanning electron micrograph of a point-contact device with a channel length of about $40 \mathrm{~nm}$ and a channel width of about $40 \mathrm{~nm}$ after device isolation. (b) An SEM image of a poly-Si film after Secco etching. GBs are clearly delineated. The size of grains ranges from $20 \mathrm{~nm}$ to $150 \mathrm{~nm}$.

*E-mail address: furuta@phy.cam.ac.uk 
shows that the geometry of the grains is columnar and perpendicular to the surface. Since the channel dimensions (width and length) of the point-contact devices were in the range of $30 \mathrm{~nm}-50 \mathrm{~nm}$, the channel may contain no GB or a few GBs at most.

Source-to-drain current-voltage $\left(I_{\mathrm{ds}}-V_{\mathrm{ds}}\right)$ characteristics were measured for more than 30 devices at from room temperature down to $19 \mathrm{~K}$. These characteristics are of two types, and typical characteristics of each type are shown in Figs. 2(a) and 2(b). Linear and nonlinear $I_{\mathrm{ds}}-V_{\mathrm{ds}}$ characteristics were obtained for Device A and Device B with the same channel length of $50 \mathrm{~nm}$ and width of $40 \mathrm{~nm}$. The resistivity for Device $\mathrm{B}$ at $V_{\mathrm{ds}} \approx 0$ in Fig. 2(b) is determined to be approximately five times larger than that for Device A (see Figs. 2(c) and 2(d)). Nonlinear $I_{\mathrm{ds}}-V_{\mathrm{ds}}$ characteristics were observed in about one-third of the fabricated devices, and the other devices showed linear characteristics. Moreover, a slight asymmetry in the $I_{\mathrm{ds}}-V_{\mathrm{ds}}$ characteristics was observed among devices with nonlinear characteristics. It should be noted that no Coulomb blockade oscillation was observed by sweeping a side-gate bias; thus, nonlinear $I_{\mathrm{ds}}-V_{\mathrm{ds}}$ characteristics are attributed to the GB potential barrier of GBs in the channel.

We analyzed the temperature dependence of the resistivity at above $200 \mathrm{~K}$ to obtain the local potential barrier height $q V_{\mathrm{B}}$ of a GB. Assuming a thermionic emission current via GBs, the current density $J$ is given by ${ }^{8,9)}$

$$
J=q n v_{\mathrm{c}} \exp \left(-\frac{q V_{\mathrm{B}}}{k_{\mathrm{B}} T}\right) \sinh \left(\frac{q V_{\mathrm{ds}}}{2 k_{\mathrm{B}} T N}\right),
$$

where $q$ is the elementary charge, $v_{\mathrm{c}}$ the collection velocity of electrons, $T$ the film temperature, $n$ the carrier concentration and $N$ the number of GBs between contacts. By fitting eq. (1) to the experimental $I_{\mathrm{ds}}-V_{\mathrm{ds}}$ curve, the value of $N$ can be obtained. The value of $N \approx 3.5$ was obtained for one of the largest devices with a channel length of $50 \mathrm{~nm}$ and width of $50 \mathrm{~nm}$. $N$ is also found to be virtually temperature independent above $200 \mathrm{~K}$. It is very likely that the number of GBs in the channel is less than four in all devices. Since the $I_{\mathrm{ds}}-V_{\mathrm{ds}}$ characteristics of the devices are determined by the electric properties of the narrow channel region, $q V_{\mathrm{B}}$ can be evaluated from the measured temperature dependence of the resistivity (see Figs. 2(c) and 2(d)) using eq. (1). The

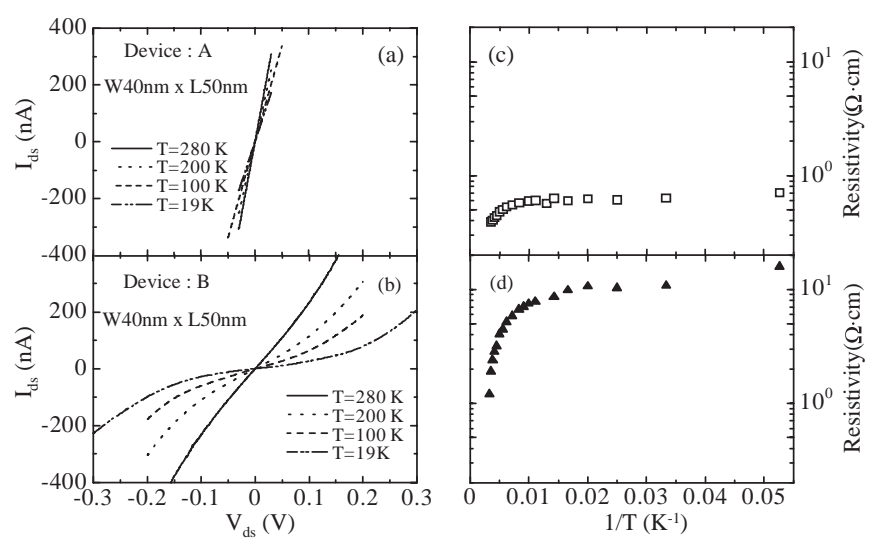

Fig. 2. (a) and (b) show source-to-drain current-voltage characteristics for Device A and Device B with a width of $40 \mathrm{~nm}$ and a length of $50 \mathrm{~nm}$ at temperatures ranging from $19 \mathrm{~K}$ to $280 \mathrm{~K}$, respectively. (c) and (d) show the temperature dependence of resistivity of Device A and Device B. distribution of $q V_{\mathrm{B}}$ for devices with nonlinear $I_{\mathrm{ds}}-V_{\mathrm{ds}}$ characteristics is shown in Fig. 3 and that for devices with linear $I_{\mathrm{ds}}-V_{\mathrm{ds}}$ characteristics is shown in the inset of Fig. 3. Note that the devices with nonlinear $I_{\mathrm{ds}}-V_{\mathrm{ds}}$ characteristics show a wide range of barrier heights from $30 \mathrm{meV}$ to $80 \mathrm{meV}$, but the devices with linear $I_{\mathrm{ds}}-V_{\mathrm{ds}}$ characteristics show a very sharp distribution with a mean value of approximately $30 \mathrm{meV}$. The small variation of $q V_{\mathrm{B}}$ for the devices with linear $I_{\mathrm{ds}}-V_{\mathrm{ds}}$ characteristics is attributed to the average potential barrier height of many GBs in the large source and drain regions outside the channel. On the other hand, the distribution of $q V_{\mathrm{B}}$ for the devices with nonlinear $I_{\mathrm{ds}}-V_{\mathrm{ds}}$ characteristics represents the GB potential barrier height in the channel. It was observed that for devices with nonlinear $I_{\mathrm{ds}}-V_{\mathrm{ds}}$ characteristics, $q V_{\mathrm{B}}$ decreases with channel width and increases with channel length. In the devices with linear $I_{\mathrm{ds}}-V_{\mathrm{ds}}$ characteristics, $q V_{\mathrm{B}}$ has no dependence on either channel length or width. This also suggests that the electron transport can be described as percolation conduction through local potential minima of GBs: $q V_{\mathrm{B}}$ can be different among adjacent GBs, and electrons can choose the GB with the lowest $q V_{\mathrm{B}}$ as an energetically favourable path. Therefore, when the channel width is increased, a GB with lower $q V_{\mathrm{B}}$ may exist in the channel. The increase in $q V_{\mathrm{B}}$ with the channel length is simply explained by the existence of multiple GBs including those with higher $q V_{\mathrm{B}}$ along the conduction path.

There are two possible causes of the variation of the potential barrier height of the GBs. One is the nonuniform intergrain distance between two adjacent grains. From highresolution XTEM observations, it was found that the intergrain thickness ranges up to $1.2 \mathrm{~nm}$ in our poly-Si films. The intergrain contains a high density of dangling bonds which create defect states near the middle of the band gap and act as trapping sites for carriers. Therefore, more trapped carriers are expected near thicker intergrain regions, resulting in a higher potential barrier height. In addition, the defect density in the intergrain can be affected by the crystal orientations of the adjacent grains. Since poly-Si films may have grains with different orientations, the local defect density in the intergrain may vary depending on the orientations of the grains nearby. The other possible cause is the difference in the active dopant concentration in the adjacent grains. During

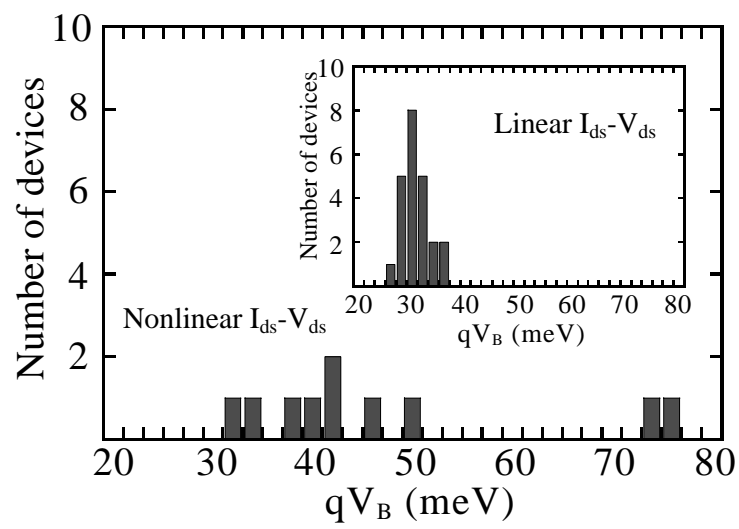

Fig. 3. Distribution of potential barrier height $q V_{\mathrm{B}}$ for the devices with nonlinear $I_{\mathrm{ds}}-V_{\mathrm{ds}}$ characteristics. The inset shows that for the devices with linear $I_{\mathrm{ds}}-V_{\mathrm{ds}}$ characteristics. 
annealing process, some phosphorus atoms segregating into GBs become electrically inactive. The percentage of these segregated atoms relative to all the phosphorus atoms will be larger for a smaller grain. As the size of individual grains is different as shown in Fig. 1(b), the density of active phosphorus atoms in the grains may also be different depending on the grain size. These imply that the local Fermi energy relative to the conduction band edge may not be the same among the adjacent grains, also leading to a nonuniform $q V_{\mathrm{B}}$. Finally, it should be noted that the small current asymmetry observed for the devices with nonlinear $I_{\mathrm{ds}}-V_{\mathrm{ds}}$ characteristics may be attributed to unbalanced local Fermi energies among the adjacent grains.

In conclusion, the local nonuniform properties of heavily doped poly-Si films were investigated using nanometer-scale point-contact devices. It was found that for devices with nonlinear $I_{\mathrm{ds}}-V_{\mathrm{ds}}$ characteristics, the effective potential barrier height $q V_{\mathrm{B}}$ of a single or few GBs ranges from about $30 \mathrm{meV}$ to $80 \mathrm{meV}$. The wide range distribution of $q V_{\mathrm{B}}$ 's may result from nonuniform local defect states.

The authors would like to thank Professor S. Oda and
Mr. K. Nishiguchi of the Tokyo Institute of Technology for their continuous support and also Dr. D. Williams of the Hitachi Cambridge Laboratory for his support in SEM observations. The authors are also grateful for the many valuable discussions with Dr. T. Shimada and Dr. G. Kawachi of Hitachi Ltd.

1) K. Nakazato, K. Itoh, H. Mizuta and H. Ahmed: Electron. Lett. 35 (1999) 848.

2) H. J. Cho, F. Nemati, P. B. Griffin and J. D. Plummer: 1998 Symp. VLSI Technology Dig. Tech. Papers, Honolulu, 1998, p. 38.

3) K. Yano, T. Ishii, T. Hashimoto, T. Kobayashi, F. Murai and K. Seki: Appl. Phys. Lett. 67 (1995) 828.

4) K. Yano, T. Ishii, T. Hashimoto, T. Kobayashi, F. Murai and K. Seki: IEEE Trans. Electron Devices 41 (1994) 1628.

5) J. W. Tringe and J. D. Plummer: J. Appl. Phys. 87 (2000) 7913.

6) ATHENA User's Manual (SILVACO International, Santa Clara, CA, 1996).

7) F. Secco d'Aragona: J. Electrochem. Soc. 119 (1972) 948.

8) T. Kamins: Polycrystalline Silicon for Integrated Circuits \& Display (Kluwer, Boston, 1998) 2nd ed.

9) J. Y. W. Seto: J. Appl. Phys. 46 (1975) 5247. 